\title{
I nostri adolescenti in fumo
}

\section{Stefania Manetti}

Pediatra di famiglia, Piano di Sorrento (Napoli)
La cosa più facile che io abbia mai fatto in vita mia è smettere di fumare: dovrei ben saperlo, perché l'ho fatto un migliaio di volte.

Mark Twain

Il titolo di questo "Osservatorio internazionale" richiama a uno studio europeo, l'European School Survey Project on Alcohol and other Drugs (ESPAD), un report con lo scopo di acquisire informazioni sull'uso e la percezione degli studenti in merito a una varietà di sostanze quali tabacco, alcol, sostanze illecite, inalanti, farmaci e nuove sostanze psicoattive.

In merito al fumo negli adolescenti cosa viene chiesto ai pediatri?

- Screenare, riguardo all'uso di sigarette elettroniche (e-cig), all'esposizione a esse e fornire nella pratica clinica consigli sulla prevenzione.

- Comunicare, attraverso un counseling adeguato, la necessità di far vivere $\mathrm{i}$ bambini e gli adolescenti in contesti liberi dal tabacco, comprese le e-cig.

- Non raccomandare le e-cig come prodotti per trattare la dipendenza da tabacco.

Secondo lo studio ESPAD Italia condotto dal CNR, nel 2018 quasi un milione e centomila studenti italiani tra i 15 e i 19 anni hanno fumato. Il consumo inizia generalmente in età adolescenziale, quasi in centomila hanno provato il fumo prima dei 12 anni. Elemento positivo è la minor facilità dichiarata dai ragazzi nel reperire le sigarette, frutto delle campagne di dissuasione e prevenzione. Tuttavia, mentre il fumo di sigarette diminuisce, cresce l'attrazione verso altri consumi della nicotina, come la sigaretta elettronica (e-cig) e quella senza combustione.

"Possiamo affermare che, nonostante il consumo di tabacco in forma di sigarette sia in costante diminuzione, non lo è affatto il consumo di nicotina. Tanto che, prendendo in considerazione il consumo di nicotina, ovvero gli studenti che riferiscono di aver fumato sigarette nell'ultimo anno e/o e-cig (sigarette elettroniche) con nicotina nella vita, la prevalenza torna vertiginosamente a salire e raggiunge livelli precedenti a quelli del 2010, anno in cui l'e-cig ha fatto la sua comparsa sul mercato italiano, con un picco pari al $46,2 \%$ nel 2015" sottolinea Sabrina Molinaro, ricercatrice CNR e responsabile scientifica dello studio ESPAD europeo. Un dato interessante è un maggiore uso in coloro che non hanno mai provato a fumare rispetto agli adolescenti fumatori occasionali e abituali.

Il problema appare simile anche oltreoceano dove, nonostante negli Stati Uniti il General Surgeon ha concluso che le sigarette elettroniche non sono sicure per i giovani, esse rappresentano a oggi la formulazione di tabacco maggiormente utilizzata tra gli adolescenti.

I giovani utilizzatori di sigarette elettroniche hanno un maggiore rischio di traslare verso l'utilizzo di sigarette con tabacco combustibile e di andare incontro a dipendenza.

La stessa Accademia americana di pediatria (AAP) raccomanda ai pediatri e ai medici di famiglia di "screenare" riguardo all'utilizzo di sigarette elettroniche tra gli adolescenti. A questa richiesta i pediatri hanno espresso il bisogno di avere maggiori risorse cliniche e strumenti per gestire l'epidemia del vaping.

L'AAP ha prodotto un percorso curriculare allo scopo di formare/informare in dettaglio sulla composizione delle sigarette elettroniche, sulle tipologie in commercio, sui loro effetti sulla salute e sull'importanza di fare prevenzione. Il materiale formativo dell'AAP (E-cigarette and Vaping Curriculum) è disponibile gratuitamente sul sito dell'AAP.

L'American Public Health Journal nel 2021 ha pubblicato un articolo dal titolo E-Cigarettes: A Public Health Threat, Not a Population Health Intervention sottolineando come non si debba parlare di un intervento di salute pubblica, bensì di una minaccia alla salute pubblica .

L'articolo evidenzia come i bilioni di dollari investiti nel tentativo di ridurre il fumo di tabacco siano stati "mandati in fumo" nel giro di pochi anni dalle sigarette elettroniche, creando delle "opportunità" senza precedenti per le nuove generazioni per poter diventare dipendenti dalla nicotina.

La nicotina è il componente psicoattivo maggiore delle soluzioni di e-cig. Spesso ci sono notevoli discrepanze tra la quantità presenti sull'etichetta e il vero contenuto della soluzione. In base alle modalità con cui il prodotto viene utilizzato l'ammontare di nicotina può superare la dose presente in una singola sigaretta tradizionale. La nicotina ha degli effetti neurotossici sullo sviluppo del cervello di un adolescente, in particolare sulle funzioni esecutive e i processi neurocognitivi ancora in via di maturazione. Quanto più precocemente un individuo usa prodotti a base di nicotina tanto maggiore è la dipendenza; di fatto la maggior parte dei fumatori adulti ha iniziato l'uso del tabacco prima dei 18 anni. Le sigarette elettroniche contengono soluzioni aromatizzate molto popolari tra i ragazzi, le ragazze e anche tra $\mathrm{i}$ bambini. Gli adolescenti percepiscono le sigarette aromatizzate come meno dannose rispetto a quelle con aromi di tabacco e si crea una percezione errata che porta a considerare queste e-cig prive di nicotina. Molte delle sostanze chimiche presenti nelle soluzioni contengono aldeidi, conosciuti come irritanti dell'apparato respiratorio, in concentrazioni tali da destare preoccupazioni. Nelle soluzioni sono presenti anche solventi necessari a produrre aerosol, come glicole propilenico o glicerolo, che sotto l'effetto del riscaldamento simulano il fumo di sigaretta. Mancano al momento dati dei loro effetti a lungo termine sulla salute. L'aerosol generato viene inalato ed espirato e una parte potrebbe essere rilasciata nell'ambiente e depositata sulle superfici. Si crea un effetto analogo a quello del fumo passivo. Di fatto nelle emissioni provenienti da e-cig sono stati riscontrate sostanze tossiche e carcinogeniche come composti organici volatili, idrocarburi policiclici, e nicotina. Sono state rinvenute sostanze metalliche e particelle di silicati a livelli maggiori rispetto alle sigarette tradizionali, risultanti dalla degradazione del rivestimento metallico usato per riscaldare la soluzione. Sono ancora pochi i dati sulla salute riguardo queste emissioni: alcuni studi suggeriscono un maggiore rischio negli adolescenti di tosse, wheezing, e riacutizzazioni di asma.

La motivazione principale che ha spinto verso l'utilizzo di e-cig è stata correlata alla necessità di smettere di fumare. Al momento mancano evidenze scientifiche a supporto, sono pochi e di piccole dimensioni gli RCT e l'evidenza sulla efficacia 
delle e-cig come aiuto allo smettere di fumare paragonata a nessun trattamento o ai trattamenti approvati dalla FDA appare insufficiente.

Studi in contesti reali hanno evidenziato che i fumatori interessati alla cessazione del fumo di sigaretta mostrano bassi livelli di successo con le e-cig paragonati a coloro che non ne hanno fatto uso. Per i fumatori incalliti le e-cig potrebbero ridurre i rischi per la salute dell'individuo se paragonati al rischio continuo di utilizzo di tabacco combustibile. Questo dato andrebbe tuttavia inserito in un maggiore contesto di salute pubblica, considerando notevole il costo in termini di salute in quanto sia negli adolescenti che negli adulti si rileva una maggiore tendenza all'utilizzo.

Dall'entrata nel 2015 delle sigarette elettroniche automatiche JUUL il mercato internazionale è stato letteralmente invaso e l'incremento del loro utilizzo tra gli adolescenti è schizzato. Il dato ancora più allarmante riguarda la scarsa o assente consapevolezza del rischio a cui si va incontro con l'utilizzo di tale prodotto.

Le sigarette elettroniche automatiche JUUL si sono poste come "un'alternativa soddisfacente al fumo di sigaretta" il cui marketing non aveva considerato la fascia adolescenziale. Una volta coinvolti i social media come Instagram, Twitter, YouTube sono stati raggiunti gli adolescenti e l'introduzione di aromi e sapori attraenti hanno portato a termine l'opera di un marketing forse apparentemente non programmato.

In una recente revisione sistematica pubblicata su Jama, le e-cig incrementano la somministrazione di nicotina e provocano potenzialmente una maggiore dipendenza. L'uso da parte dei giovani e dei giovani adulti può essere attribuito all'accettabilità dal punto di vista sociale, alle caratteristiche di praticità del prodotto, al marketing aggressivo sui social e anche al design che le rende facilmente camuffabili. Appare estremamente urgente trovare idonee strategie per prevenire l'utilizzo così diffuso di queste sigarette.
Le sigarette elettroniche JUUL contengono una frazione piuttosto bassa di nicotina libera nel liquido del pod e nell'aerosol paragonate ad altri tipi di sigarette elettroniche (5-6\% in media rispetto al 13-95\%), ma un elevato contenuto di nicotina nella sua forma di sale benzoato. Questa formulazione assicura una diffusione di alte dosi di nicotina a valori di $\mathrm{Ph}$ bassi, apparentemente meno forte rispetto a contenuti di nicotina riscontrati in altre marche di sigarette elettroniche, ma il consumatore è portato a una più profonda inalazione. I livelli urinari di 38 adolescenti che hanno utilizzato questo tipo di sigarette era maggiore rispetto al livello riscontrato in adolescenti fumatori di tabacco da sigarette normali.

Nel 2018 l'FDA ha richiesto a eBay di rimuovere le vendite online di JUUL, tuttavia altri venditori hanno continuato a vendere online evitando di menzionarne il nome commerciale.

Nel 2017 il volume dei tweet associati a JUUL è aumentato di 17 volte rispetto al 2016 e i video su YouTube hanno superato le centomila visualizzazioni. Un'analisi di 9.077 utenti che seguono l'account Twitter di JUUL ha stimato che 1'81\% dei follower aveva un età compresa tra 13 e 20 anni. Pochissimi, tra i post analizzati, quelli che menzionavano i rischi per la salute.

Le sigarette elettroniche automatiche contengono livelli maggiori di nicotina. I rischi per la salute devono essere chiaramente esplicitati agli adolescenti, in particolare i maggiori rischi di andare incontro a dipendenza . Le caratteristiche del prodotto, compresi i contenuti di nicotina, dovrebbero essere ben definiti. Esistono online ancora molte possibilità di acquisto e questi rivenditori dovrebbero essere banditi così come i canali diretti di marketing che arrivano agli adolescenti.

Solide evidenze sottolineano come i giovani adolescenti che utilizzano le sigarette elettroniche sono significativamente più esposti all'uso di sigarette tradizionali.

Nel 2010, il ministero della Salute ha chiesto ai produttori di sigarette elettroniche di evidenziare, su tutti i prodotti, la concentrazione di nicotina, di apporre i necessari simboli di tossicità e di evidenziare la frase TENERE LONTANO DALLA PORTATA DEI BAMBINI su tutti i prodotti in vendita . Nel 2011 il ministero della Salute adotta l'ordinanza di divieto di vendita di sigarette elettroniche contenenti nicotina ai minori di 16 anni. Nel 2013 il divieto viene innalzato a 18 anni.

Nel 2013 viene emanata anche una prima regolamentazione dell'uso delle e-cig nei luoghi pubblici e della loro pubblicità. Le sigarette elettroniche vengono equiparate ai prodotti del tabacco rispetto alla tassazione, alla pubblicità, alla vendita nelle tabaccherie, alla tutela della salute dei non fumatori. Nel settembre 2013 un provvedimento legislativo vieta l'utilizzo delle e-cig nei locali chiusi delle istituzioni scolastiche statali, ma è meno rigido sulla pubblicità, che viene consentita con dei limiti al fine di tutelare i minorenni (decreto legge n. 104 del 2013). Nello stesso anno viene cancellato il divieto di utilizzo della sigaretta elettronica nei luoghi pubblici. Rimane il divieto nelle scuole, comprese le aree all'aperto di pertinenza degli istituti (legge n. 128 del 2013).

doc.manetti@gmail.com

www.aap.org

Juhyun Lee S, Rees VW, Yossefy N, et al. Youth and Young Adult Use of Pod-Based Electronic Cigarettes From 2015 to 2019: A Systematic Review. JAMA Pediatr. 2020 Jul 1;174(7):714-720.

Jenssen BP, Walle SC. E-Cigarettes and Similar Devices.Pediatrics.2019Feb;143(2):e20183652. Gee RE, Boles WR, Smith DG. E-Cigarettes: A Public Health Threat, Not a Population Health Intervention. Am J Public Health. 2021 Feb;111(2):224-226.

National Academies of Sciences, Engineering, and Medicine, Health and Medicine Division, Board on Population Health and Public Health Practice, Committee on the Review of the Health Effects of Electronic Nicotine Delivery Systems. Public Health Consequences of E-Cigarettes. The National Academies Press, 2018. 